\title{
A novel scheme for the reporting of adverse drug reactions
}

\author{
A Clarkson, E Ingleby, I Choonara, P Bryan, P Arlett
}

\begin{abstract}
Background-The safety of medicines used in children is of considerable public interest, yet available data to monitor the safety of medicines in children is limited. Aims-To raise awareness and stimulate reporting of adverse drug reactions (ADRs) in children in the Trent region.

Methods-A pilot Paediatric Regional Monitoring Centre (PRMC) has been established in the Trent region. The scheme operates as an extension of the UK's spontaneous reporting scheme, the Yellow Card Scheme run by the Medicines Control Agency and the Committee on Safety of Medicines. Proactive interventions including a monthly reminder letter and presentations to staff in the identified hospitals have been made.

Results-During the first year of the PRMC, 95 reports were received from the Trent region compared to 40 for the previous year. Twenty four of these reports were for medicines used "off label". The 95 reports involved 105 drugs and 171 suspected ADRs. Twenty six of the ADRs (15\%) were considered medically significant.

Conclusions-The number of ADR reports from the Trent region has increased considerably in the first year of the scheme. The results show that intensive education and promotion of ADR reporting can result in a major increase in reporting. This initiative will increase our knowledge about the safety of medicines used to treat children and so help protect public health.

(Arch Dis Child 2001;84:337-339)
\end{abstract}

Child Health

(University of

Nottingham),

Derbyshire Children's

Hospital, Uttoxeter

Road, Derby

DE22 3NE, UK

A Clarkson

E Ingleby

I Choonara

Medicines Control

Agency, Market

Towers, 1 Nine Elms

Lane, London

SW8 5NQ, UK

P Bryan

P Arlett

Correspondence to:

Prof. Choonara

imti.choonara@

nottingham.ac.uk

Accepted 30 November 2000
Paediatric Regional Monitoring Centre (PRMC) has been established. The scheme runs as an extension of the UK's spontaneous ADR reporting scheme-the Yellow Card Scheme. We describe the findings after the first 12 months of the scheme.

Methods

The PRMC was established in October 1998 in the Academic Division of Child Health, University of Nottingham at the Derbyshire Children's Hospital. The scheme is funded jointly by the Medicines Control Agency (MCA) and Trent NHS, providing the funding for a paediatric clinical research pharmacist and a part time data entry clerk. Before the PRMC was established, yellow cards were sent directly to the MCA.

East Anglia and Oxford was chosen as a comparative region with a similar population of children and with no CSM regional monitoring centre. This is an important consideration as $\mathrm{ADR}$ reporting is encouraged by the regional monitoring centres and therefore may have affected both the usefulness of the scheme and the ability to compare the two regions.

Twenty hospitals were identified in the Trent region with paediatric patients (16 years and under, including neonates). Paediatricians, paediatric pharmacists, paediatric anaesthetists, and paediatric surgeons who practised in each of the hospitals were identified using membership registers or by contacting the relevant hospital department directly. Each person identified was included on the mailing list.

Each month a reminder letter, an update letter, and a spare Yellow Card were sent to each person on the mailing list. The reminder letter was intended to stimulate reporting for certain drugs and reactions. It was requested that reports be submitted for certain black triangle drugs, serious reactions to any drugs, and patients with Stevens-Johnson syndrome where a drug was suspected as the cause. There was also a list of specific reactions to certain drugs with the aim of increasing the number of reports for these and thereby enabling any predisposing factors leading to ADRs to be identified. These included skin reactions to lamotrigine and topical local anaesthetics, arrhythmias to cisapride, visual field defects with vigabatrin, systemic adverse reactions to inhaled or nasal corticosteroids, and any adverse reactions to leukotriene antagonists.

This information was sent either by post or electronically. Electronic and telephone reporting of ADRs was encouraged. The scheme has been promoted by way of presentations to the staff at the hospitals involved in the scheme as well as regional presentations. 
Table 1 Types of adverse drug reactions

\begin{tabular}{lc}
\hline Type of reaction & Number of reactions \\
\hline Localised skin reaction & 48 \\
Rash & 17 \\
Abdominal pain & 6 \\
Convulsions & 5 \\
Diarrhoea & 4 \\
Pyrexia & 4 \\
Adrenal suppression & 3 \\
Alopecia & 3 \\
Flatulence & 3 \\
Headache & 3 \\
Nausea & 3 \\
Abnormal behaviour & 2 \\
Cough & 2 \\
Growth retarded & 2 \\
Haematuria & 2 \\
Pallor & 2 \\
Upper limb oedema & 2 \\
Vomiting & 2 \\
Miscellaneous & 58 \\
Total & 171
\end{tabular}

The reporter sends the completed yellow card to the PRMC and the information is forwarded to the MCA. If any information is missing from the card it is requested at this time from the reporter along with any other information that might be useful. A literature search is carried out to find out more information regarding the reported $\mathrm{ADR}$. The results of this literature search, along with details of previous reports to the MCA/CSM are then sent to the reporter with an acknowledgement letter. A report was deemed medically significant if it was fatal, potentially life threatening, or disabling. Each report was also assessed as to whether the suspected drug had been used in a licensed manner by consulting the Summary of Product Characteristics or by contacting the manufacturer by phone.

\section{Results}

In the first 12 months, 95 yellow cards were received by the PRMC. During the same time period 18 yellow cards were submitted directly to the MCA by hospital health professionals in the Trent region for suspected ADRs in children. In the preceding 12 months, when the yellow cards were sent directly to the MCA, there had only been 40 yellow cards received from hospitals for suspected ADRs to children in the Trent region, and 22 in the year before that. In comparison, 26 yellow cards were received for children in hospital in East Anglia and Oxford during the first 12 months of the scheme.

In the 95 yellow cards received by the PRMC, there were 171 suspected adverse drug reactions-that is, 1.8 reactions per card. There were 105 suspected drugs on the 95 yellow cards - that is, 1.1 per card. In total, 54 different medicines were involved in the 95 reports. Table 1 shows the type of reactions reported. Four types of drug were responsible for more than $50 \%$ of the yellow cards received (see table 2). One third of the yellow cards involved local skin reactions to topical anaesthetics. Over half the ADRs reported involved drugs not mentioned in the monthly letter.

The majority of reports were from hospital doctors in paediatric medicine (59\%). Over one third of reports were from paediatric phar-
Table 2 Groups of drugs responsible for adverse drug reactions

\begin{tabular}{lc}
\hline Class of drug & Number of reports \\
\hline Topical local anaesthetics & \\
Antibiotics & 35 \\
Anticonvulsants & 13 \\
Vaccines & 10 \\
Corticosteroids (inhaled and nasal) $^{\star}$ & 10 \\
Pancreatic enzyme supplements & 4 \\
Antiemetics & 4 \\
Opioids & 2 \\
Miscellaneous & 2 \\
Total & 25 \\
\end{tabular}

$\star$ Reports for these groups of drugs were specifically requested in monthly reminder letters.

Table 3 Medically significant adverse drug reactions

\begin{tabular}{lll}
\hline Age & Suspected drug & Reaction \\
\hline $3 \mathrm{mth}$ & $\begin{array}{l}\text { DTP Hib and } \\
\text { polio }\end{array}$ & $\begin{array}{l}\text { Depressed level of } \\
\text { consciousness }\end{array}$ \\
$8 \mathrm{y}$ & Fluticasone & Adrenal suppression \\
$7 \mathrm{y}$ & Fluticasone & Adrenal suppression \\
$2 \mathrm{y}$ & Interferon alfa & $\begin{array}{l}\text { Motor development } \\
\text { impaired }\end{array}$ \\
$1 \mathrm{y}$ & Diamorphine & Stevens-Johnson syndrome \\
$1 \mathrm{wk}$ & Baclofen & Neonatal convulsion \\
$11 \mathrm{y}$ & Vigabatrin & Visual disturbance \\
$11 \mathrm{mth}$ & Bupivacaine & Supraventricular tachycardia \\
& & Ventricular fibrillation \\
$11 \mathrm{y}$ & Cephradine & Anaphylactic reaction \\
$9 \mathrm{y}$ & Carbamazepine & Hypovolaemia \\
& & Renal failure, acute \\
$8 \mathrm{y}$ & Cyclosporin & Colitis \\
$4 \mathrm{mth}$ & DTP vaccine & Convulsions \\
$13 \mathrm{y}$ & Fluticasone nasal & Benign intracranial \\
& spray & hypertension \\
$6 \mathrm{y}$ & Diclofenac & Haematemesis \\
$15 \mathrm{y}$ & Lansoprazole & Neutropenia \\
$4 \mathrm{y}$ & Fluticasone & Adrenal suppression \\
& & Hypoglycaemic convulsion \\
$4 \mathrm{mth}$ & DTP Hib & Convulsions \\
$1 \mathrm{day}$ & Atracurium & Acute circulatory failure \\
& & Apnoea \\
& & Convulsions \\
& & Disseminated intravascular \\
& & coagulation \\
& & Hypoglycaemia \\
& & Metabolic acidosis \\
\hline & &
\end{tabular}

$\star^{\star}$ Reports for these drugs were specifically requested in monthly reminder letters.

macists (36\%). Other groups involved in the reporting included anaesthetists and immunologists. Many nurses detected ADRs but are currently not recognised reporters of ADRs under the yellow card scheme other than for certain vaccines.

The percentage of yellow cards received for children from both community and hospital reporters in comparison to the total number of yellow cards received for patients of all ages historically has been $9 \%$ in Trent (data for 1996-97 and 1997-98). The figure for the UK nationally during the first 12 months of the scheme excluding Trent was also $9 \%$. The figure for Trent region in the first 12 months of the scheme was $15 \%$, confirming that the PRMC resulted in a significant increase in reporting. This increase has come from hospital reports as the level of general practitioner (GP) reporting has remained constant.

The licensing status of the reported drugs was reviewed. Twenty four of the 95 reports had at least one suspected drug that was judged to be used off label.

Twenty six of the 171 ADRs (15\%) were considered to be medically significant; these 
were associated with 18 of the 95 yellow card reports (table 3). Five of these 18 reports related to a drug specifically mentioned in the monthly letter.

\section{Discussion}

The existence of the PRMC resulted in an increase of reports when compared with previous data from the same region and in comparison to a region with a similar population of children. Also the number of yellow cards received for children, as a percentage of the total number of yellow cards received was higher in Trent than the national average. This increase in yellow cards suggests an increased awareness of ADRs. This is illustrated by the medically significant ADRs, where over two thirds of the yellow cards involved drugs not mentioned on the monthly letter.

Adverse drug reactions in children have usually been reported as case reports, but occasionally have involved prospective studies of drug toxicity. ${ }^{6}$ Drug surveillance in children is crucial, as many medicines that are used have not been formally tested in clinical trials. It is for this reason that many medicines used in children are used off label. It was reassuring to see that the majority of reports we received related to medicines that appear to be both licensed and used in accordance with the manufacturer's instructions. Previous studies in children have suggested that $25-46 \%$ of drug prescriptions are either unlicensed or off label. ${ }^{34}$ However, only $25 \%$ of the yellow cards received by the PRMC involved a drug that was thought to be used off label. It is possible that the data entered on to the yellow card were insufficient to recognise that a medicine was being used off label or that ADRs to off label and unlicensed medicines are less likely to be reported.

Several of the reports were brought to the attention of a pharmacist by a nurse. Nurses are currently not able to report suspected ADRs to the yellow card scheme, so a pharmacist has to report it on their behalf. Studies have shown the value of nurses reporting ADRs, ${ }^{7}$ although this has not previously been reported for children. Topical local anaesthetics were responsible for the largest number of reports, many of which were detected by nurses. Reactions to topical local anaesthetics were requested in the reminder letter. The large number of yellow cards for topical local anaesthetics may also be because a topical reaction is easy to identify and describe. The other groups of drugs responsible for many ADR reports included antibiotics, which are prescribed extensively in children in hospital; anticonvulsants, where ADRs are known to be frequent ${ }^{8}$; and vaccines, which are widely prescribed for children.

GPs were not included in the scheme initially as we wished to address the issue of under reporting of paediatric ADRs in hospital patients. A systematic review found that approximately $9 \%$ of children experience an
ADR while in hospital when monitored prospectively. Very few of these reactions are reported spontaneously. The number of reports received from GPs in the Trent region has remained approximately the same in the first year of the scheme as it has for the previous two years. This suggests that the reporting of paediatric ADRs does not appear to have "shifted" from GPs to hospital practice as a result of the PRMC.

There was only one report received for a neonate. This is surprising as it has previously been shown that between $11 \%$ and $30 \%$ of neonates in intensive care will suffer at least one ADR. ${ }^{9}$ This suggests that ADRs in neonates in Trent are still grossly under reported. This is probably caused by a combination of factors, including the lack of awareness of the possibility of an ADR and the difficulty in recognising ADRs in critically ill neonates. It is to be hoped that this issue can be tackled in the second year of the scheme.

Previous studies of ADRs in children have either concentrated on specialised groups of patients or tertiary centres. We have shown that ADRs occur in children admitted to all types of centre, be they tertiary centres or smaller district general hospitals. The types of ADRs described through this surveillance scheme show that the range of ADRs in children may be as broad as that in adults. The number of ADR reports from the Trent region has increased considerably in the first year of the scheme. The results show that intensive education and promotion of $\mathrm{ADR}$ reporting can result in a significant increase in the number of yellow cards received. The PRMC has achieved its objectives of increasing awareness and the reporting of ADRs in children. There is, however, insufficient data at present to establish the optimal method for the Regional Monitoring Centre contributing to increased awareness of ADRs in children on a national scale. It is to be hoped that more information about drug toxicity in children will result in safer use of medicines and the prevention of severe drug toxicity.

The Paediatric Regional Monitoring Centre is funded by the MCA and Trent NHS

1 British Paediatric Association. Licensing medicines for children. London: BPA, May 1996.

2 Brown EG, Choonara I. The safety of medicines used in children. International fournal of Pharmaceutical Medicine 1998;12:285-8.

3 Turner S, Longworth A, Nunn AJ, Choonara I. Unlicensed and off label drug use in paediatric wards: prospective study. BMF 1998;316:343-5.

4 Conroy S, Choonara I, Impicciatore P, et al. Survey of unlicensed and off label drug use in paediatric wards in European countries. $B M \mathcal{F} 2000 ; 320: 79-82$.

5 Impicciatore P, Choonara I, Clarkson A, et al. Prospective surveillance of adverse drug reactions in pediatric in/outpatients: a systematic review and meta-analysis. Submitted.

6 Choonara I, Gill A, Nunn AJ. Drug toxicity and surveillance in children. Br f Clin Pharmacol 1996;42:407-10

7 Morrison-Griffiths S, Pirmohamed M. Specialist nurse Morrison-Griffiths S, Pirmohamed M. Specialist nurse
reporting of adverse drug reactions. Professional Nurse reporting of adverse drug reactions. Professional Nurse 2000,15:300-5.

8 Choonara A. Anticonvulsant toxicity in paediatric outpatients. Br f Clin Pract 1988;42:21-4

9 Bonati M, Marchetti F, Zullini MT, et al. Adverse drug reactions in neonatal intensive units. Adverse Drug React Acute Poisoning Rev 1990;9:103-18. 